\title{
Descriptive Experience Sampling as a Psychological Method
}

\section{Charles Fernyhough and Ben Alderson-Day}

\begin{abstract}
This chapter outlines the practice of descriptive experience sampling (DES), a methodology with which Hubbub has experimented. Interdisciplinary DES experiments and workshops run during Hubbub's residency brought collaborators together to explore the profoundly varied ways in which the resting state can be conceptualized, and the different forms that perspectives on aspects of inner experience might take.
\end{abstract}

Keywords Descriptive experience sampling - fMRI - Inner experience . Introspection $\cdot$ Mind wandering $\cdot$ Resting state

Researchers have become increasingly interested in characterizing psychological and neural states in the absence of any specific external stimulation. It is now widely recognized that a brain that is not engaged with any particular task demonstrates highly organized patterns of activity, resonating with the much longer established idea that a 'resting' mind is anything but still. Although different terminologies have arisen to describe psychological states that are not focused on specific tasks (such as stimulus

C. Fernyhough $(\square)$ B B. Alderson-Day

Durham University, Durham, United Kingdom

e-mail: c.p.fernyhough@durham.ac.uk; benjamin.alderson-day@durham.ac.uk

(C) The Author(s) 2016

F. Callard et al. (eds.), The Restless Compendium,

DOI 10.1007/978-3-319-45264-7_6 
independent thought, daydreaming, etc.), research has cohered around the dominant term mind wandering as a designator of the varied experiences of a resting mind. ${ }^{i}$

Despite this growth of interest in mind wandering and the resting state, there have been few attempts to provide rich descriptions of the phenomenology (or 'what it is like' qualities) of resting cognition. One reason has been anxieties about the reliability of introspection, ranging from William James' concerns about the reliance of introspection on memory, ${ }^{1}$ to critiques about the unreliability of participants' self-reports on the causes of their actions. ${ }^{2}$ In attempts to avoid the methodological pitfalls of introspection, participants are typically asked, through questionnaires, to report on what was in their experience during the few minutes of the so-called 'resting-state' scan (a procedure where participants are asked to lie in the brain scanner without performing any particular task). Such catch-all reports are unlikely to provide detailed information on the complex, multi-modal and dynamic patterning of the stream of consciousness, which is likely to vary considerably in its qualities from individual to individual. Another approach has been to present participants with random probes and ask them to report on their experience at the moment of the probe. Drawbacks of that method include its likelihood of disturbing the very experiences and neural activations under investigation, and the highly constrained nature of the response options available (e.g. making a simple choice between whether one's attention was focused on the task or not). Such methodological limitations have meant that key questions about the phenomenology of resting-state cognition, such as the extent to which it has a verbal quality, remain largely unexamined. ${ }^{3}$

An alternative approach to characterizing a resting mind is to elicit more detailed descriptions of very specific moments of inner experience. However, extended and unguided introspective reports are open to various kinds of bias, including the effects of self-generalizations and self-theoretical attitudes. One method that acknowledges and tries to obviate such risks is descriptive experience sampling (DES). In this method, pioneered by the psychologist Russell Hurlburt of the University of Nevada, Las Vegas, the participant wears a small beeper attached to their clothing which delivers a series of random beeps to an earpiece worn in the ear. On hearing the beep, the participant's task is to make brief notes about what was in their experience in the moment

\footnotetext{
${ }^{i}$ See Chaps. 4 and 5.
} 
just before the beep's onset. The participant then takes these notes along the next day to an in-depth interview with DES investigators, who interview the participant in great detail about the moments of experience captured in the previous day's sampling. At the heart of the method is a concern that asking people to report on their own experiences is as likely to elicit preconceptions about the nature of experience at least as much as it elicits any accurate reports on it. ${ }^{\text {ii }}$ Crucially, DES involves an iterative method in which participants are assumed to find the task difficult at the outset and accordingly not to produce accurate descriptions. Over repeated cycles of sampling and interviewing, however, participant and investigators establish a common framework for talking about that specific participant's experiences, leading to increasing accuracy and focus of beep descriptions. ${ }^{4}$

Our involvement with DES research began when we started a collaboration with Hurlburt and the neuroscientist Simone Kühn at the Max Planck Institute for Human Development in Berlin. Over the course of five weeks, we trained five volunteers as DES participants, first in their natural environment (going about their daily lives in Berlin) and next in the magnetic resonance imaging (MRI) scanner. This represented the first time DES had been used in conjunction with neuroimaging. ${ }^{5}$ Each participant underwent nine separate resting-state scans of 25 minutes each, resulting in a total of 225 minutes of resting-state functional magnetic resonance imaging ( $\mathrm{fMRI}$ ) data for each participant. In addition, each participant reported on 36 randomly sampled DES beeps collected while they were undergoing the resting-state scan. We also asked participants to complete a standard questionnaire on their experience in the resting state, the ReSQ. ${ }^{6}$

The results of this study have been described by Hurlburt and colleagues. ${ }^{7}$ We found that people were generally quite consistent in what they reported in the resting state. There were considerable individual differences, for example in the reporting of inner speech, and those differences were consistent across sampling sessions. We also found considerable divergence between what people reported in DES and their responses on the ReSQ. This supports Hurlburt's contention that questionnaires generally assess participants' preconceptions about their experience at least as much as their actual experience: that is, people tend to fill in questionnaires on the basis of what they think their experience is like, rather than

\footnotetext{
ii See also Chap. 11.
} 
how it actually is. ${ }^{\text {iii }}$ Although our sample size was too small to allow the drawing of firm conclusions, it was interesting that the gap between DES and ReSQ responses narrowed as participants became more expert in the sampling process.

These endeavours led to the richest body of qualitative data currently available on the phenomenology of resting-state cognition. Here are a couple of examples:

Lara is looking at the edges of the scanner mirror - left bottom corner, and sees two of them, layering. Simultaneously she is hearing herself say, to no one in particular, 'I really want to talk to you'. The voice is recognized to be her own, expressed in her own natural way; however, the vocal characteristics are not of her own voice but of some female voice that she doesn't recognize. The wrongness of the vocal characteristics was noted only retrospectively - at the moment of the beep, experientially, Lara simply hears herself talking. She knows who the 'you' is in this sentence, but the sentence is not directed to that person. She is also seeing her hands.

Susan innerly sees the actress Sigourney Weaver in a cryogenic tank from the movie Alien. She sees Sigourney's face from above, below the glass window of the tank - the rest of Sigourney's body is vaguely or blurrily present. Mostly Susan is searching for the word used in the movie: Cryogenic chamber, cryogenic tank, etc., waiting for the right word to appear. This is primarily a state of suspended animation, waiting for the word - she does not see or hear pieces of words, etc.

Our study design also allowed us to address some specific questions about the verbal nature of resting-state cognition. Covert self-directed speech (known as inner speech) is known to be reasonably frequent in the resting state, with one study reporting that 90 per cent of participants engaged in it. ${ }^{8}$ In addition to classifying DES beeps according to whether they involved inner speech or not, we incorporated a standard inner speech elicitation paradigm, in which participants had to generate covert utterances in the scanner. Spontaneous and elicited inner speech related to strikingly different patterns of neural activation, casting doubt on the reliability of elicitation paradigms for this and other forms of mental experience. ${ }^{9}$ In other words, asking people to have a particular experience in the brain scanner is no guarantee that they will do so, or at least

iii See Chap. 8. 
that what results will bear much resemblance to the naturally occurring phenomenon.

One strength of DES is its capacity to describe states of experience that may be experienced by only a minority of participants - or which may even be unique to one individual. ${ }^{\text {iv }}$ Such idiosyncratic states of consciousness are by definition very difficult to capture in self-report questionnaires. While DES studies often describe phenomena occurring in the common modalities of visual imagery, inner speech, unsymbolized thought, sensory awareness and feelings (what Hurlburt has termed the five frequent phenomena), the method also allows the creation of new categories if these are the best ways of describing a specific participant's experiences. For example, DES studies have shown that people's descriptions of inner speech take two forms: inner speaking and inner hearing. In inner speaking, the participant has the experience of being the producer of the speech that is experienced. In inner hearing, by contrast, the sense is more of being the recipient of the produced speech. One of our participants, Lara, showed this less common form of inner hearing, and it occurred frequently enough in her DES samples for us to compare these two forms of inner speech in a single-case design. ${ }^{10}$

DES is not a perfect method, and there are several grounds for caution. First, it has been argued that the act of observation inevitably changes the event itself: either in the sampling moment, or as a result of the series of interviews that follow the sampling session. ${ }^{11}$ Second, bridging the delay between the collection of samples and the interview relies on participants being able to adequately remember enough about their experiences to report them accurately. Hurlburt has argued that variations in the time between beep and interview have made little difference to the reports he collects, as long as the delay is less than 24 hours Also important is the fact that notes on the sampled moment are taken immediately, with the iterative process aiming to ensure increasingly greater fidelity and focus of note-taking as the sampling process unfolds. However, such issues around whether observing and reporting may affect moments of experience remain a lingering concern, and one that can likely only be unpicked through further empirical research.

The various strengths and weaknesses of DES have been discussed in depth by Hurlburt elsewhere, especially with the sceptical philosopher Eric

\footnotetext{
iv See Chap. 15.
} 
Schwitzgebel. ${ }^{12}$ Notably, while Schwitzgebel is doubtful about the possibility of any kind of accurate introspection, he concludes that DES is nevertheless the most high-fidelity method for investigating inner experience currently available. Indeed, many of the criticisms levelled at DES could also be directed towards other, more superficial self-report methods: if introspection is a problem for DES, then a much larger swathe of psychological research faces similar problems.

Notwithstanding such concerns, we argue that DES represents an alternative methodology for investigating inner experience that can complement and enhance existing neuroscientific methods. ${ }^{13}$ The richness of the data it provides also allows for current models of mind wandering and resting-state cognition to be tested against one another. One focus of our research in Hubbub has been on drawing on collaborators' expertise in mind wandering and the resting state to put such models to the test. In ongoing work, we are recoding the resting-state sampling vignettes from our Berlin study according to whether they appear to be primarily internally directed, or initiated by events in the external environment. This allows us to test a cognitive neuroscientific model of how the brain switches between internally and externally stimulated thought, opening such models up to new kinds of qualitative data. ${ }^{14}$

Future research will also extend the investigation into overlap between resting-state cognition and inner speech. In terms of its neural bases, inner speech activates a particular network of regions primarily in the left hemisphere of the brain. One question of interest is how language plugs in to the dynamic system that coordinates switching between on-task and taskfree cognition. ${ }^{15}$ Another focus is the idea that verbal mind wandering involves a more abstract form of inner speech, while more voluntary inner speech has a more concrete or expanded form. ${ }^{16}$ This exemplifies one of the ways in which a focus on new kinds of qualitative data can open up novel research questions about the resting mind and brain. ${ }^{v}$

\section{Notes}

1. William James, The Principles of Psychology (London: Macmillan, 1901).

2. Richard E. Nisbett and Timothy D. Wilson, 'Telling More than We Can Know: Verbal Reports on Mental Processes', Psychological Review 84, no. 3 (1977): 231-59.

${ }^{v}$ See Chaps. 5 and 7. 
3. Ben Alderson-Day and Charles Fernyhough, 'Inner Speech: Development, Cognitive Functions, Phenomenology, and Neurobiology', Psychological Bulletin 141, no. 5 (2015): 931-65.

4. Charles Fernyhough, The Voices Within: The History and Science of How We Talk to Ourselves (London: Profile Books/Wellcome Collection, 2016), chap. 3.

5. Simone Kühn et al., 'Inner Experience in the Scanner: Can High Fidelity Apprehensions of Inner Experience Be Integrated with fMRI?', Frontiers in Psychology: Cognitive Science 5 (2014): 1393.

6. Pascal Delamillieure et al., 'The Resting State Questionnaire: An Introspective Questionnaire for Evaluation of Inner Experience during the Conscious Resting State', Brain Research Bulletin 81, no. 6 (2010): 565-73.

7. Russell T. Hurlburt et al., 'What Goes on in the Resting-State? A Qualitative Glimpse into Resting-State Experience in the Scanner', Frontiers in Psychology: Cognitive Science 6 (2015): 1535.

8. Delamillieure et al., 'The Resting State Questionnaire'.

9. Russell T. Hurlburt et al., 'Exploring the Ecological Validity of Thinking on Demand: Neural Correlates of Elicited vs. Spontaneously Occurring Inner Speech', PLoS One 11, no. 2 (2016): e0147932.

10. Kühn et al., 'Inner Experience in the Scanner'.

11. M. Perrone-Bertolotti et al., 'What Is That Little Voice inside My Head? Inner Speech Phenomenology, Its Role in Cognitive Performance, and Its Relation to Self-Monitoring', Behavioural Brain Research 261 (2014): 220-39.

12. Russell T. Hurlburt and Eric Schwitzgebel, Describing Inner Experience?: Proponent Meets Skeptic (Cambridge, Mass.: MIT Press, 2007).

13. Ben Alderson-Day and Charles Fernyhough, 'More than One Voice: Investigating the Phenomenological Properties of Inner Speech Requires a Variety of Methods', Consciousness and Cognition 24 (2014): 113-14.

14. Jonathan Smallwood, Kevin Brown, Ben Baird, Jonathan W. Schooler, 'Cooperation...' between the default mode network and the frontal-parietal network in the production of an internal train of thought', Brain Research 1428 (January 5, 2012): 60-70.

15. Fernyhough, The Voices Within.

16. Perrone-Bertolotti et al., 'What Is That Little Voice inside My Head?'; Alderson-Day and Fernyhough, 'Inner Speech'.

\section{FURTHER READING}

Smallwood, Jonathan, and Jonathan W. Schooler 'The Science of Mind Wandering: Empirically Navigating the Stream of Consciousness.' Annual Review of Psychology 66 (January 2015), 487-518. 
Charles Fernyhough is a writer of fiction and non-fiction, and an academic at Durham University (Department of Psychology). His latest non-fiction book is The Voices Within: The History and Science of How We Talk to Ourselves (Profile/ Wellcome Collection, 2015). He is an Associate Director of Hubbub.

Ben Alderson-Day is a psychologist and cognitive neuroscientist at Durham University. His work has included research on hallucination experiences in clinical and non-clinical populations, and executive function and categorization in autism spectrum disorders.

\footnotetext{
(c) (i) This chapter is distributed under the terms of the Creative Commons Attribution 4.0 International License (http://creativecommons.org/ licenses/by/4.0/), which permits use, duplication, adaptation, distribution and reproduction in any medium or format, as long as you give appropriate credit to the original author(s) and the source, a link is provided to the Creative Commons license and any changes made are indicated.

The images or other third party material in this chapter are included in the work's Creative Commons license, unless indicated otherwise in the credit line; if such material is not included in the work's Creative Commons license and the respective action is not permitted by statutory regulation, users will need to obtain permission from the license holder to duplicate, adapt or reproduce the material.
} 